\title{
Fast Detection Method in Cooperative Cognitive Radio Networks
}

\author{
Zhengyi Li, Lin Liu, and Chi Zhou \\ Department of Electrical and Computer Engineering, Illinois Institute of Technology, Chicago, IL 60626, USA \\ Correspondence should be addressed to Chi Zhou, zhouc@ece.iit.edu
}

Received 1 December 2009; Revised 13 May 2010; Accepted 8 June 2010

Academic Editor: Hsiao Hwa Chen

Copyright () 2010 Zhengyi Li et al. This is an open access article distributed under the Creative Commons Attribution License, which permits unrestricted use, distribution, and reproduction in any medium, provided the original work is properly cited.

\begin{abstract}
Cognitive Radio (CR) technology improves the utilization of spectrum highly via opportunistic spectrum sharing, which requests fast detection as the spectrum utilization is dynamic. Taking into consideration the characteristic of wireless channels, we propose a fast detection scheme for a cooperative cognitive radio network, which consists of multiple CRs and a central control office. Specifically, each CR makes individual detection decision using the sequential probability ratio test combined with Neyman Pearson detection with respect to a specific observation window length. The proposed method upper bounds the detection delay. In addition, a weighted $K$ out of $N$ fusion rule is also proposed for the central control office to reach fast global decision based on the information collected from CRs, with more weights assigned for CRs with good channel conditions. Simulation results show that the proposed scheme can achieve fast detection while maintaining the detection accuracy.
\end{abstract}

\section{Introduction}

In the traditional management of licensed spectrum, users usually pay and have the exclusive access of spectrum with a certain level of Quality of Service (QoS) guarantee. On one hand, the spectrum is getting more and more crowded as the number of wireless devices increases drastically. However, on the other hand, the utilization of spectrum at any given time is low. Figure 1 shows a measurement of $30 \mathrm{M}-3 \mathrm{GHz}$ spectrum utilization. We can see that a lot of spectrum bands are vacant. Therefore, it would be efficient to allow unlicensed users to share spectrum with licensed users by using a vacant frequency band.

Cognitive Radio technology is developed to utilize these white spaces intelligently [1, 2]. FCC Spectrum Policy Task Force published a new spectrum management policy, open access or license exempted model, in 2002, to allow unlicensed user to use the opportunistic spectrum. As the transition from analog to digital television is complete, there are vacant channels (white spaces) in every media market [3]. Accordingly, the FCC announced a Notice of Proposed Rule Making (NPRM) on 13 May 2004, which proposed "to allow unlicensed radio transmitters to operate in the broadcast TV spectrum at locations where that spectrum is not being used". Seen as the secondary user, the cognitive radio (CR) must avoid interfering with primary user (PU), that is, licensed user, while sharing the licensed band with the PU. Therefore, cognitive radio needs to sense the spectrum to detect the existence of PU, identify the white spaces of spectrum, and adapt its transmission to one of the white spaces to avoid interfering with PU.

Detecting the vacant bands of the spectrum is the very first step but very crucial in Cognitive Radio technology. There are three major digital signal processing techniques that could be used to detect the existence of PU: matched filtering, energy detection, and cyclostationary feature detection $[4,5]$. Among those, energy detector has been used widely due to its simplicity and easy implementation [6]. As a radio device, a single CR may suffer severe shadowing or multipath fading with respect to primary transmitter so that it cannot detect the existence of PU even in its vicinities. In addition, there exists a hidden-node problem, in which a $\mathrm{CR}$ may be too far from the PU to detect the existence, but close to the primary receiver to interfere with the reception if transmited. Cooperative sensing provides a solution to the challenges mentioned above [7, 8]. In cooperative sensing, multiple cognitive radios cooperate to reach an optimal global decision by exchanging and combining individual local sensing results. Allowing multiple CRs to cooperate, cooperative sensing can increase the detection probability, reduce the detection time, and achieve the diversity gain [9$18]$. 


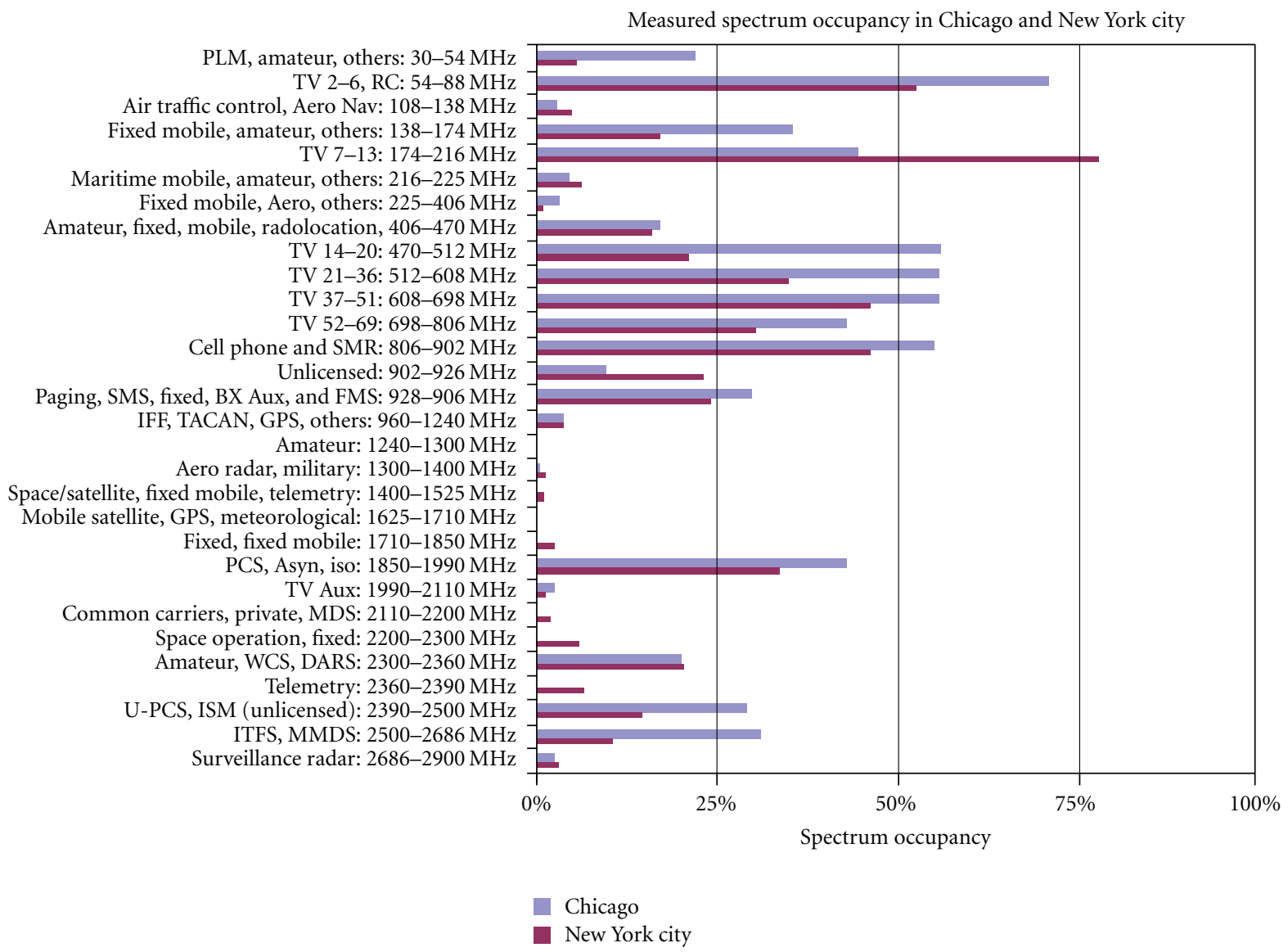

Figure 1: A measurement of 30M-3GHz spectrum utilization.

Due to the fading and noisy wireless channel, a large number of samples are needed for accurate detection. However, the spectrum utilization is dynamic, which requests fast detection to enable opportunistic sharing. In this paper, we propose a fast detection scheme in a cooperative cognitive radio network, which consists $N$ CRs and a central control office. Each CR makes individual detection decision and then forwards its decision and the average signal to noise radio (SNR) to the central control office, which will make a global detection decision based on the collected data from CRs in the network. Then the central control office broadcasts the global detection decision to all the CRs. The proposed scheme consists of two folds: the first is to propose Sequential Probability Ratio Test (SPRT) method with a truncated window to upper bound the detection time at individual $\mathrm{CR}$, while satisfying the detection accuracy requirements; the second is to propose a weighted $K$ out of $N$ fusion rule, which assigns more weights for CRs with good channel conditions, at the central control office to speed up the global decision making by using less number of individual decisions. Simulation results show that the proposed scheme can achieve fast detection while maintaining the detection accuracy.

The remaining contents are organized as follows. In Section 2, we discuss the system model. Section 3 presents the proposed fast detection scheme. In Section 4, simulation results are presented. In the end, we give the conclusion.

\section{System Model}

We consider a cognitive network, which consists of $N$ CRs and a central control office. Each CR is equipped with an energy detector to individually detect the existence of PU by measuring the received SNR. Once the detection decision is reached by a $\mathrm{CR}$, the $\mathrm{CR}$ transmits its decision along with the average received SNR to the central control office for global decision making. Serving as a fusion center, the central control office applies some fusion rule to its collected data and reach the global detection decision. Then the central control office broadcasts the global detection decision to all the CRs. Widely adopted Ad hoc On-demand Distance Vector (AODV) routing protocol [19] is used over a default clear channel for information exchange between the CRs and the central control office. The default channel may be selected among several predetermined channels.

In this paper, we use the log-normal shadowing path loss model:

$$
P_{r}(d)=P_{0}\left(d_{0}\right)+10 \cdot n \cdot \log \left(d_{0} \backslash d\right)+X(0, \delta),
$$

where $P_{r}(d)$ is the received signal power at distance $d, P_{0}\left(d_{0}\right)$ is the received power at the reference point $d_{0}, n$ is the path loss exponent, and $X(0, \delta)$ is normal shadowing random variable with zero mean and $\delta$ standard variance in $\mathrm{dB}$.

We summarize the major notations which will be used in the paper in Table 1. 


\section{Fast Detection Scheme}

In this section, a fast detection scheme is proposed and discussed in details. SPRT with truncated window is proposed for individual detection, followed by a weighted $K$ out of $N$ fusion rule for the central control office to reach quick global decision.

3.1. Individual Detection. Fast and accurate individual detection is a must. To achieve desired detection accuracy, multiple samples need to be taken due to the time-varying wireless links. One approach is to take a certain amount of samples and then make a one-time decision, such as Neyman Pearson method [20]. Another approach is sequential detection, that is, the detection decision criterion will be checked whenever one new sample is taken, such as Sequential Probability Ratio Test (SPRT) [21]. Neyman Pearson method has a fixed detection delay, while SPRT usually takes less-detection time on average but may take long delay though with small probability. We propose to combine these two approaches together to take advantages of the two. Specifically, we propose to impose a truncated window to SPRT so that the detection delay is bounded. When the number of the samples is less than the window size, original SPRT is used to do the sequential detection. If the sequential detection cannot reach decision when the window size is reached, Neyman Pearson method will be used to make the final decision. The proposed SPRT with truncated window achieves smaller detection delay compared with SPRT and Neyman Pearson method.

3.1.1. Sequential Probability Ratio Test. We define two hypotheses, specified as follows:

$$
\begin{gathered}
H_{0} \text { : the primary user does not exist, } \\
H_{1} \text { : the primary user does exist. }
\end{gathered}
$$

When a CR observes a new sample from energy detector, it will compute the cumulative sum of the log-likelihood ratio. We assume all samples are i.i.d. Let $y_{i}$ be the received power from the $i$ th observed sample, and then the log-likelihood ratio for the sample is

$$
l\left(y_{i}\right)=\ln \frac{p d f\left(y_{i} \mid H_{1}\right)}{p d f\left(y_{i} \mid H_{0}\right)} .
$$

When $H_{0}$ is true, that is, the PU does not exist, $y_{i}$ is just the noise power. When $H_{1}$ is true, that is, the PU does exist, $y_{i}$ is the received signal power plus the noise power. Let noise $(i)$ be the AWGN noise for the $i$ th sample with zero mean and variance $\delta_{n}^{2}$. Then $y_{i}$ is normal distributed

$$
\begin{aligned}
& \text { If } H_{0} \text { is true: } y_{i}=\operatorname{noise}(i) \sim \operatorname{Normal}\left(0, \delta_{n}^{2}\right), \\
& \text { If } H_{1} \text { is true: } y_{i}=u+\operatorname{noise}(i) \sim \operatorname{Normal}\left(u, \delta_{n}^{2}\right),
\end{aligned}
$$

where $u$ is the signal power. Therefore, the log-likelihood ratio for the sample is

$$
l\left(y_{i}\right)=\frac{u y_{i}}{\delta_{n}^{2}}-\frac{u}{2 \delta_{n}^{2}}
$$

TAble 1: Notation table.

\begin{tabular}{ll}
\hline$\lambda_{0}, \lambda_{1}:$ & two stopping bounds in SPRT \\
$P_{m, \mathrm{sprt}}:$ & miss-detection probability in SPRT \\
$P_{d, \mathrm{spr}}:$ & detection probability in SPRT \\
$P_{f, \mathrm{spr}}:$ & false alarm probability in SPRT \\
$P_{d, \mathrm{NP}}:$ & detection probability in NP \\
$P_{f, \mathrm{NP}}:$ & false alarm probability in NP \\
$P_{d d}:$ & detection probability in SPRT-TW \\
$P_{f f}:$ & false alarm probability in SPRT-TW \\
$P\left(H_{0}\right):$ & statistic probability of $H_{0}$ \\
$P\left(H_{1}\right):$ & statistic probability of $H_{1}$ \\
$w:$ & window size \\
$I_{i}:$ & individual decision \\
$\alpha_{i}:$ & assigned weight value \\
$\mathrm{SNR}_{i}:$ & received SNR of the $i$ th CR \\
$d_{0}:$ & reference distance \\
$n:$ & path loss exponent \\
$P_{0}\left(d_{0}\right):$ & received power at reference distance \\
$P_{\mathrm{rc}}:$ & confidence probability \\
\hline
\end{tabular}

The cumulative sum of the log-likelihood ratio can be written in the sequential way as

$$
L\left(Y_{i}\right)=L\left(Y_{i-1}\right)+l\left(y_{i}\right)
$$

where

$$
L\left(Y_{0}\right)=0
$$

It can be also written as

$$
L\left(Y_{i}\right)=\sum_{k=1}^{i} l\left(y_{k}\right) .
$$

According to (5), we have

$$
L\left(Y_{i}\right)=\sum_{k=1}^{i} l\left(y_{k}\right)=\frac{u}{\delta_{n}^{2}} \sum_{k=1}^{i} y_{k}-\frac{i u}{2 \delta_{n}^{2}} .
$$

The cumulative sum of the log-likelihood ratio will be compared with two stopping bounds, $\lambda_{0}$ and $\lambda_{1}$, to make decision. When the cumulative sum $L\left(Y_{i}\right)$ is larger than $\lambda_{1}$, we accept $H_{1}$ hypothesis and the detection process stops. If the cumulative sum $L\left(Y_{i}\right)$ is less than $\lambda_{0}$, we accept $H_{0}$ hypothesis and the detection process also stops. However, when $L\left(Y_{i}\right)$ lies between these two bounds, a new sample will be taken and the cumulative sum will be updated and compared with the bounds. The sequential detection process continues until it stops.

These two stopping bounds are set to satisfy the required miss-detection probability $P_{m \text {,sprt }}$ and false alarm probability $P_{f, \text { sprt }}$. They can be approximated as [21]

$$
\begin{aligned}
& \lambda_{0} \approx \ln \frac{P_{m, \mathrm{sprt}}}{1-P_{f, \mathrm{sprt}}}, \\
& \lambda_{1} \approx \ln \frac{1-P_{m, \mathrm{sprt}}}{P_{f, \mathrm{sprt}}} .
\end{aligned}
$$


Let $T$ be the detection time. We could obtain expected detection time:

$$
\begin{aligned}
& E\left(T \mid H_{1}\right)=\frac{\left(1-P_{m, \mathrm{sprt}}\right) \lambda_{1}+P_{m, \mathrm{sprt}} \lambda_{0}}{E\left(l\left(y_{i}\right) \mid H_{1}\right)}, \\
& E\left(T \mid H_{0}\right)=\frac{\left(1-P_{f, \mathrm{sprt}}\right) \lambda_{0}+P_{f, \mathrm{sprt}} \lambda_{1}}{E\left(l\left(y_{i}\right) \mid H_{0}\right)} .
\end{aligned}
$$

3.1.2. Proposed SPRT with Truncated Window. The sequential detection process is random and may take a very long time before it stops. In order to put an upper bound on the detection time, we impose a truncated window with size $w$ to SPRT. If SPRT cannot stop within $w$ samples, instead of taking more samples, we apply Neyman Pearson (NP) method to reach immediate decision while achieving certain false alarm probability $P_{f, \mathrm{NP}}$ and detection probability $P_{d, \mathrm{NP}}$ with $w$ samples.

The proposed SPRT with truncated window (SPRT-TW) scheme is summarized as follows:

$$
\begin{aligned}
& L\left(Y_{i}\right) \geq \lambda_{1} \text { : Accept } H_{1}, \\
& L\left(Y_{i}\right) \leq \lambda_{0} \text { : Accept } H_{0},
\end{aligned}
$$

$\lambda_{0}<L\left(Y_{i}\right)<\lambda_{1}$ and $i<w$ : Continue sampling,

$\lambda_{0}<L\left(Y_{i}\right)<\lambda_{1}$ and $i=w$ : Apply NP method.

Therefore, the individual detection probability $P_{d d}$ and the individual false alarm probability $P_{f f}$ for the proposed SPRT-TW could be written as, according to Bayes' Rule,

$$
\begin{aligned}
& P_{d d}=P_{d, \mathrm{NP}}(w) \cdot P(T>w)+P_{d, \mathrm{sprt}} \cdot P(T \leq w), \\
& P_{f f}=P_{f, \mathrm{NP}}(w) \cdot P(T>w)+P_{f, \mathrm{sprt}} \cdot P(T \leq w),
\end{aligned}
$$

where $P(T>w)$ is the probability that the CR does not reach a decision within window size $w$ samples and $P(T \leq w)$ is the probability that the $\mathrm{CR}$ reaches a decision within window size $w$. According to the rule of total probability, the two probabilities can be expressed as

$$
\begin{aligned}
P(T>w)= & P\left(T>w \mid H_{1}\right) \cdot P\left(H_{1}\right) \\
& +P\left(T>w H_{0}\right) \cdot P\left(H_{0}\right), \\
P(T \leq w)= & P\left(T \leq w \mid H_{1}\right) \cdot P\left(H_{1}\right) \\
& +P\left(T \leq w \mid H_{0}\right) \cdot P\left(H_{0}\right)
\end{aligned}
$$

where $P\left(H_{0}\right)$ and $P\left(H_{1}\right)$ are statistical probabilities for the two hypothesis and

$$
\begin{gathered}
P\left(T>w \mid H_{1}\right)=\prod_{i=1}^{w} P\left(\lambda_{0}<L\left(Y_{i}\right) \leq \lambda_{1} \mid H_{1}\right), \\
P\left(T>w \mid H_{0}\right)=\prod_{i=1}^{w} P\left(\lambda_{0}<L\left(Y_{i}\right) \leq \lambda_{1} \mid H_{0}\right), \\
P\left(T \leq w \mid H_{1}\right)=1-P\left(T>w \mid H_{1}\right), \\
P\left(T \leq w \mid H_{0}\right)=1-P\left(T>w \mid H_{0}\right) .
\end{gathered}
$$

From (9), $L\left(Y_{i}\right)$ is the sum of normally distributed random variables. Therefore, $L\left(Y_{i}\right)$ follows normal distribution

$$
\begin{aligned}
& \text { If } H_{0} \text { is true: } L\left(Y_{i}\right) \sim \operatorname{Normal}\left(-b, a^{2} i \delta_{n}^{2}\right), \\
& \text { If } H_{1} \text { is true: } L\left(Y_{i}\right) \sim \operatorname{Normal}\left(a u i-b, a^{2} i \delta_{n}^{2}\right),
\end{aligned}
$$

where

$$
\begin{aligned}
& a=\frac{u}{\delta_{n}^{2}}, \\
& b=\frac{i u^{2}}{2 \delta_{n}^{2}} .
\end{aligned}
$$

The expected detection delay for SPRT-TW could be obtained:

$$
\begin{aligned}
E\left(T \mid H_{1}\right)= & 1 \cdot P\left(T \leq 1 \mid H_{1}\right) \\
& +\sum_{i=2}^{w} i \cdot P\left(T>i-1 \mid H_{1}\right) P\left(T=i \mid H_{1}\right) \\
& +w \cdot P\left(T>w \mid H_{1}\right) P_{d, \mathrm{NP}}(w), \\
E\left(T \mid H_{0}\right)= & 1 \cdot P\left(T \leq 1 \mid H_{0}\right) \\
& +\sum_{i=2}^{w} i \cdot P\left(T>i-1 \mid H_{0}\right) P\left(T=i \mid H_{0}\right) \\
& +w \cdot P\left(T>w \mid H_{0}\right) P_{f, \mathrm{NP}}(w),
\end{aligned}
$$

where

$$
\begin{aligned}
& P\left(T>w \mid H_{1}\right)=1-P\left(\lambda_{0}<L\left(Y_{w}\right) \leq \lambda_{1} \mid H_{1}\right), \\
& P\left(T>w \mid H_{0}\right)=1-P\left(\lambda_{0}<L\left(Y_{w}\right) \leq \lambda_{1} \mid H_{0}\right) .
\end{aligned}
$$

Based on (13)-(17), the expected delay could be obtained easily.

3.2. Weighted $K$ out of $N$ Fusion Rule. Data fusion is a technique used to efficiently combine the data for decision making. Due to its simplicity and effectiveness, $K$ out of $N$ fusion rule has been widely used in many applications including cognitive radio $[7,22]$. We could also apply the $K$ out of $N$ fusion rule in the central control office to reach the global detection decision. Similar to [23], the global decision rule could be specified as

$$
\begin{aligned}
& \sum_{N} I_{i} \geq K: \text { Accept } H_{1}, \\
& \sum_{N} I_{i}<K: \text { Accept } H_{0},
\end{aligned}
$$

where $I_{i}$ is the indicator of individual detection decision for $\mathrm{CR}_{i} . I_{i}=1$ if $\mathrm{CR}_{i}$ accepts $H_{1}$ and $I_{i}=0$ if $\mathrm{CR}_{i}$ accepts $H_{0}$.

The above $K$ out of $N$ fusion detection rule implies that each data has the same credibility as others by simply adding the individual detection decisions together. However, this is not true in wireless communication systems. For example, suppose two CRs correctly detect the existence of the PU with 
one CR located very close to the PU and the other located far away from the PU. The nearby CR receives strong signal and quickly detects the PU, while the far-away $\mathrm{CR}$ receives very weak signal and takes much longer time to reach the decision. Obviously, the detection decision from the nearby $\mathrm{CR}$ is more reliable, which is not taken into account in the original $K$ out of $N$ fusion rule. Therefore, we propose a weighted $K$ out of $N$ fusion rule by assigning bigger weight to the CR with good signal reception (i.e., good channel condition). Then the global decision rule is specified as

$$
\begin{aligned}
& \sum_{N} \alpha_{i} I_{i} \geq K: \text { Accept } H_{1}, \\
& \sum_{N} \alpha_{i} I_{i}<K: \text { Accept } H_{0},
\end{aligned}
$$

where $\alpha_{i}$ is the weight for individual decision of $\mathrm{CR}_{i}$. There are many ways to design the weight $\alpha_{i}$ to reflect the credibility of individual decision. In this paper, as an example, we design the weight as a linear function of received SNR

$$
\alpha_{i}=A \cdot \mathrm{SNR}_{i}+B
$$

where $A$ and $B$ are some constants.

In the weighted $K$ out of $N$ fusion rule, the individual detection decisions under good channel conditions are given more weights in the global decision making. Therefore, the global decision making requests a small number of CRs if those CRs have good channel condition or a large number of CRs if they have bad channel condition. Since those CRs with good channel condition also have smaller detection time using SPRT-TW and consequently their decisions arrive at the central control office faster, the global detection time can be reduced when most CRs have good channel condition.

\section{Simulation Results}

In this section, we first consider the individual detection performance for each $\mathrm{CR}$, and then evaluate the weighted $K$ out of $N$ fusion rule for global detection. For the log-normal shadowing path loss model, the shadowing random variable $X(0, \delta)$, adds the randomness to the results, which complicates the illustration and insight discussion. Therefore, in the simulation for the individual detection, we first consider the log-distance path loss model without fading and then generalize it to slow fading scenario. The simulation results in the no fading scenario help understand the whole innovative fast detection scheme. Throughout simulation, we set the following parameters: the noise follows normal distribution with zero mean and the noise power set as $-120 \mathrm{dBm}$; the path loss exponent $n=4 ; P_{0}\left(d_{0}\right)$ is set as $20 \mathrm{dBm}$; reference distance is set $d_{0}=1 \mathrm{~m}$; the statistic probabilities $P\left(H_{0}\right)=P\left(H_{1}\right)=0.5$.

4.1. Individual Detection Scenario 1: No Fading. With no fading, the path loss model is simplified to the log-distance path loss model and the average received signal power is

$$
u=P_{r}(d)=P_{0}\left(d_{0}\right)+10 \cdot n \cdot \log \left(d_{0} \backslash d\right) .
$$

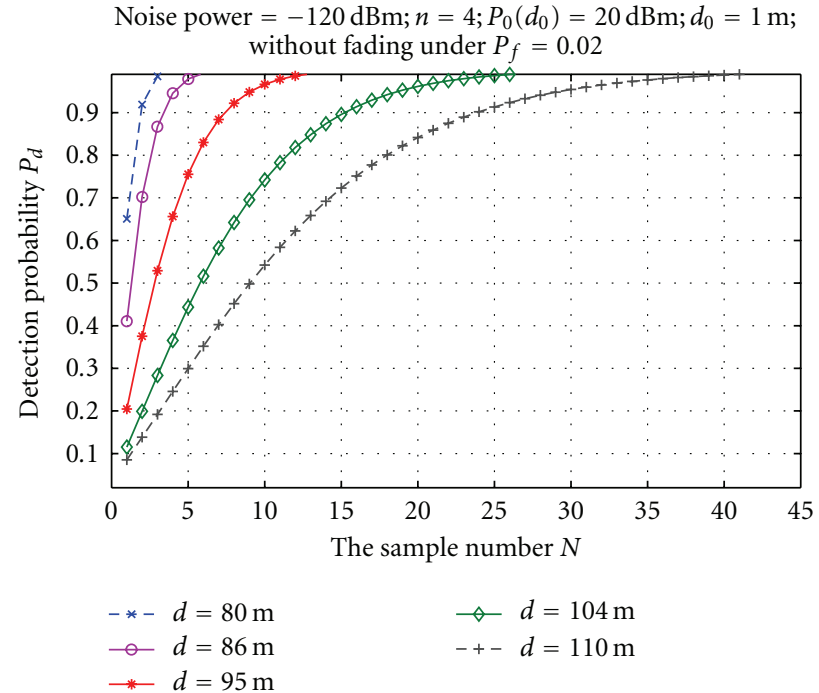

FIgURE 2: Traditional NP method: under fixed $P_{f, \mathrm{NP}}=0.02$.

CRs with longer distance to the $\mathrm{PU}$ receive weaker signal power, therefore, the distance can be used to represent the received signal strength or the received SNR.

Note that the detection performance depends on the window size $w$ and received signal power $u$. Intuitively, the larger the window size is, the better the detection performs; the stronger the received signal power $u$ is, the better the detection performs. We first examine the performance of traditional Neyman Pearson (NP) method when varying the number of samples (i.e., the window size $w$ in SPRTTW). We fix the false alarm probability for NP method as 0.05 . Figure 2 shows how the detection probability of NP method varies with the number of samples for CRs at different distance from the PU. It is shown that the detection probability increases as the number of samples increases and the CR with smaller distance (i.e., stronger signal) achieves higher detection probability for any given number of samples. As shown in Figure 2, the traditional NP method needs up to 42 samples to reach 0.99 detection probability for CRs at the distance $110 \mathrm{~m}$.

We set the two stopping bounds of SPRT based on the miss-detection probability $P_{m \text {,sprt }}=0.01$ and false alarm probability $P_{f \text {,sprt }}=0.02$ and simulate the proposed SPRTTW. Figure 3 shows how the detection probability $P_{d d}$ of SPRT-TW varies with the window size for CRs with different distance. Figure 3 has the same trend as Figure 2. Shown in Figure 3, the CR at distance $110 \mathrm{~m}$ (received SNR is $58.343 \mathrm{~dB}$ ) takes most window size $w=18$ to meet 0.99 detection probability while the CR located at $d=80 \mathrm{~m}$ (received SNR is $63.8764 \mathrm{~dB}$ ) only needs 2 samples on average to reach the same detection probability. Compared with the NP method, SPRT-TW takes less samples to reach the same detection probability (e.g., 42 samples for NP method and 18 samples for SPRT-TW at the same CR at $d$ $=110 \mathrm{~m}$ ).

Figure 4 shows the corresponding false alarm probability $P_{f f}$ of SPRT-TW as the window size varies. It is shown that 


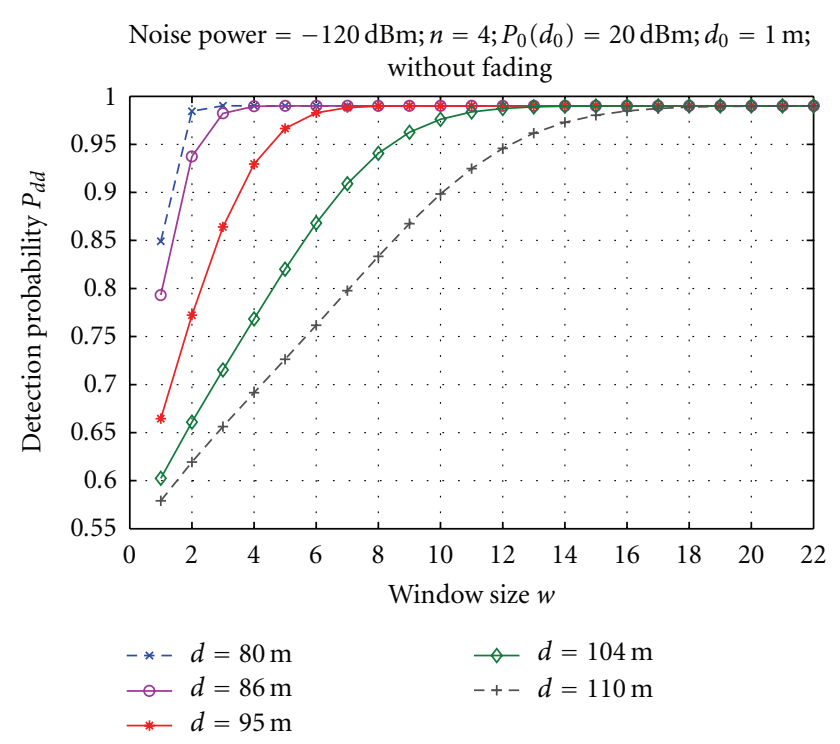

Figure 3: Detection probability $P_{d d}$ versus Window size.

$P_{f f}$ decreases with the window size and the CR at the smaller distance achieves smaller $P_{f f}$ for any given window size. Shown in Figure 4 , the CR at $d=110 \mathrm{~m}$ needs window size $w=20$ to reach 0.02 false alarm probability, which takes only 4 window size for the CR at $d=80 \mathrm{~m}$.

From Figures 3 and 4, we see that the window size needs to be carefully selected to meet the desired detection performance. In addition, the selection of window size also depends on the channel condition (i.e., received SNR). In order to minimize the detection delay, we need to select the minimum window size $w$ that can meet the performance requirements.

We further compare the detection delay among NP method, SPRT, and our proposed SPRT-TW. Figure 5 shows that the NP method needs the longest delay and our proposed SPRT-TW has smallest delay. The simulation results validate that our SPRT-TW is indeed a fast detection scheme compared to NP method and original SPRT.

4.2. Individual Detection Scenario 2: Slow Fading. In this section, we consider the log-normal shadowing path loss model, shown in (1). Because of the random fading factor $X \sim N(0, \delta(\mathrm{dB}))$, we define a confidence probability Prc,

$$
\operatorname{Prc}=P\left(P_{r}(d)>\varepsilon\right),
$$

where the $P_{r}(d)$ is received power and $\varepsilon$ is the power threshold. Prc describes how much confidence we have when the receive power is stronger than the power threshold. In the simulation, we set $\delta=8 \mathrm{~dB}$ for shadowing and $n=4$ for path loss exponent.

We set the threshold power as $-56.1236 \mathrm{dBm}$ and plot how the confidence probability varies with distance in Figure 6. It is shown that the confidence probability decreases with distance. To achieve 0.9 confidence level, the CR has to locate closer than $45 \mathrm{~m}$ from the PU. Without fading, however, the CR can locate at far as $d=80 \mathrm{~m}$ to receive

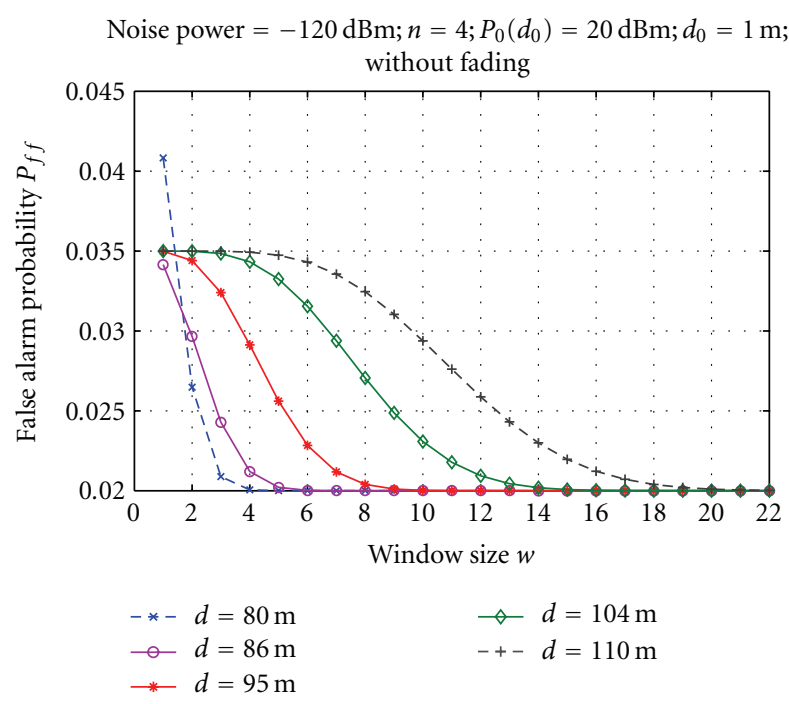

Figure 4: False alarm probability $P_{f f}$ versus Window size

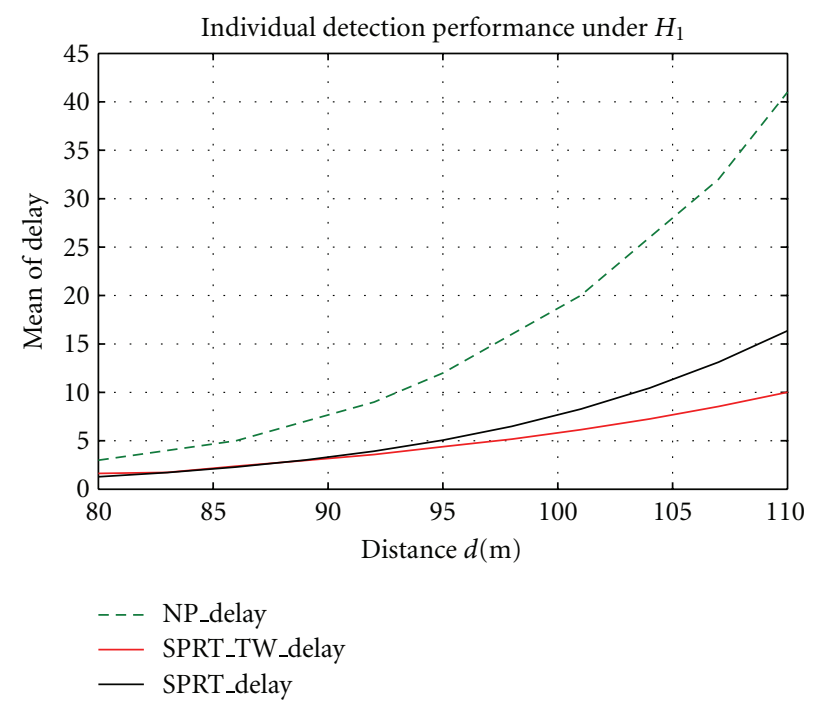

FIgURE 5: Delay performance Comparison.

$-56.1236 \mathrm{dBm}$ power. Therefore, the $\mathrm{CR}$ at $d=45 \mathrm{~m}$ under fading condition should use the same window size as the CR at $d=80$ without fading. We also try various threshold power and identify the distances for no fading scenario and fading scenario which use the same window size to achieve similar detection performance. The results are shown in Table 2 under 0.9 confidence level. It is shown that the fading has negative impacts for the detection performance.

4.3. Weighted $K$ out of $N$ Fusion Rule. In this section, we compare our proposed weighted $K$ out of $N$ fusion rule with the original $K$ out of $N$ fusion rule. From [23], we know that $K$ is usually chosen as $N / 2$ to minimize the total error probability, shown in Figure 7.

We randomly generate $N=100$ CRs according to a uniform distribution at the distance from the PU ranging 


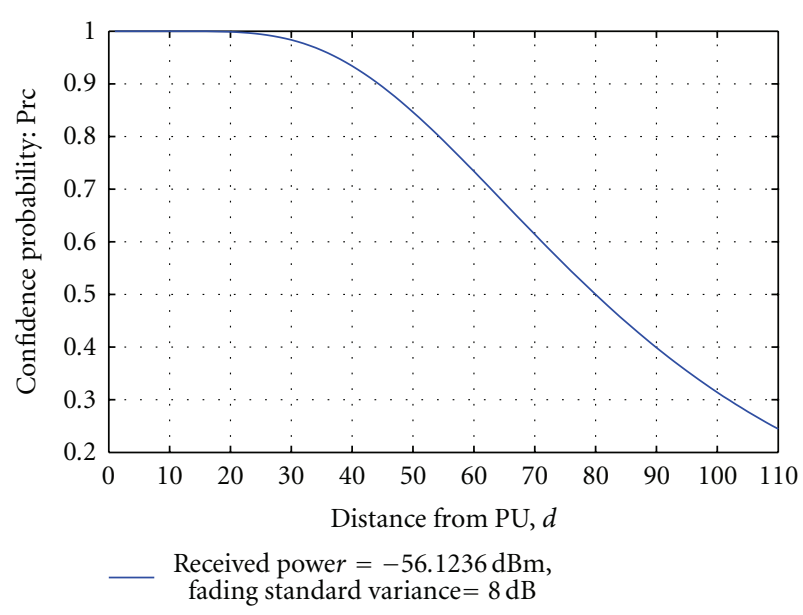

Figure 6: Confidence probability under threshold $-56.1236 \mathrm{dBm}$ versus Distance $d$.

TABle 2: Comparing the distance from PU under fading and no fading.

\begin{tabular}{lcc}
\hline Threshold power $(\mathrm{dBm})$ & No fading & Slow fading \\
\hline-56.1236 & $80(\mathrm{~m})$ & $45(\mathrm{~m})$ \\
-57.3799 & $86(\mathrm{~m})$ & $47(\mathrm{~m})$ \\
-59.1089 & $95(\mathrm{~m})$ & $52(\mathrm{~m})$ \\
-60.6813 & $104(\mathrm{~m})$ & $57(\mathrm{~m})$ \\
-61.6557 & $110(\mathrm{~m})$ & $62(\mathrm{~m})$ \\
\hline
\end{tabular}

from $80 \mathrm{~m}$ to $110 \mathrm{~m}$. We use the linear weight function to assign the weight to each CR. For fair comparison, we set the values $A$ and $B$ such as the expectation of weights is equal to 1 but the variance of the weights can vary. Figures 8 and 9 show the weight assigned for the CRs at different distance with the mean weight as 1 but the weight variance as 0.5 and 0.02 , respectively. It is shown that the nearby CR is assigned with higher weight compared to the far-away CR due to the good channel condition. In addition, the weights for CRs at different distances differ more when the weight variance is larger.

We compare the minimum number of individual decisions needed to reach the global decision. We pick $K=$ $N / 2=50$ so that the total error probability can be minimized in the original $K$ out of $N$ Fusion Rule. For the original fusion rule, each individual decision is treated the same with weight 1, therefore, minimum 50 individual detection decisions with all positive detections are needed. In the weighted fusion rule, the individual decision is treated differently. To minimize the number of individual decisions, we need to include the CRs with the best channel conditions (i.e., the CRs closest to the PU). Example results are shown in Table 3. It is shown that the weighted fusion rule needs less minimum number of individual decisions. In addition, the more the weight variance is, the less number of individual decisions the fusion needs. Since those decisions come from the CRs with best channel conditions and consequently arrive at the

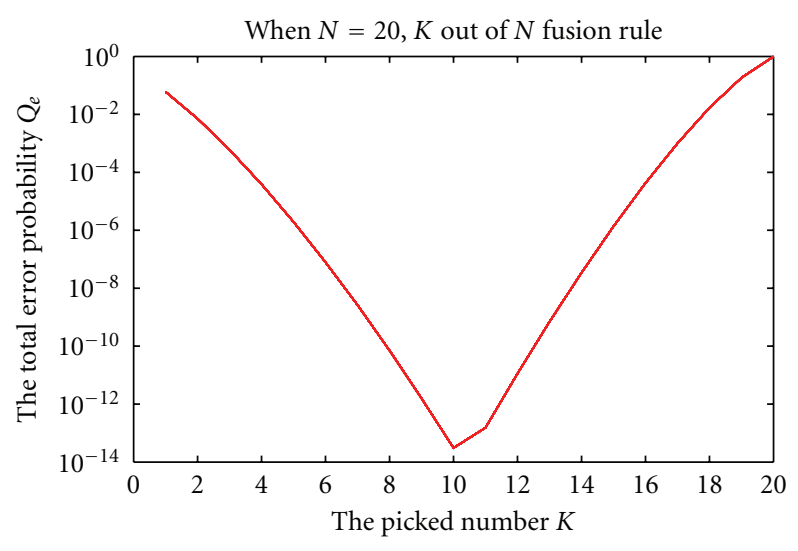

FIgURE 7: Total error probability versus value $K$ when $N=20$.

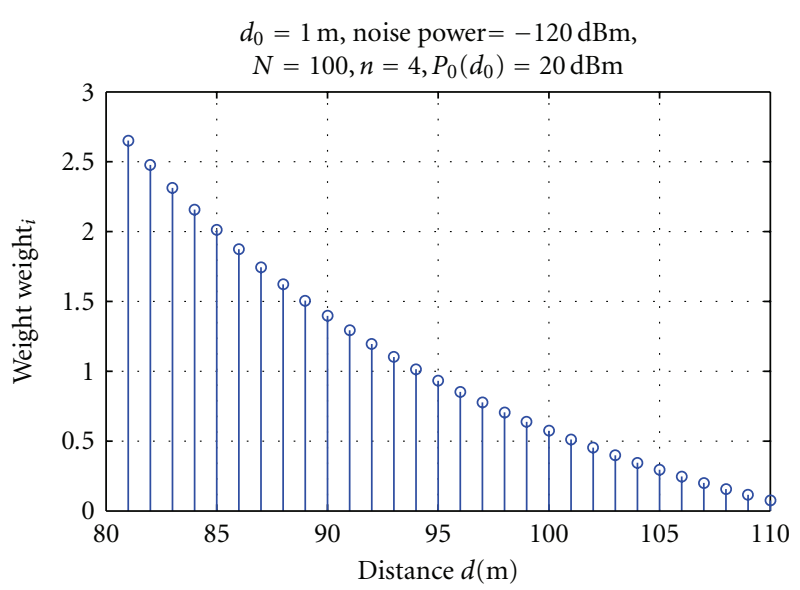

FIGURE 8: Assigned weight for CRs at different distance when weight variance $=0.5$.

TABLE 3: Comparing the original and the weighted fusions.

\begin{tabular}{lcc}
\hline$N=100$ & Original fusion & Weighted fusion \\
\hline variance $=0$ & $M=50$ & $M=50$ (same as the original $)$ \\
variance $=0.02$ & $M=50$ & $M=44$ \\
variance $=0.5$ & $M=50$ & $M=26$ \\
\hline
\end{tabular}

central control office quickest, the global decision can be reached quickly without waiting for more decisions.

\section{Conclusion}

In this paper, we have proposed a fast detection scheme, SPRT-TW for individual detection and weighted $K$ out of $N$ fusion rule for global detection, for cooperative cognitive radio networks. It is shown that the proposed SPRT-TW takes the least detection time compared with traditional NP detection method and the original SPRT and the weighted fusion rule in general takes less numbers of individual decisions (consequently faster) to reach the global decision compared to the original fusion rule. Our scheme takes into consideration the characteristic of wireless channels. For the 


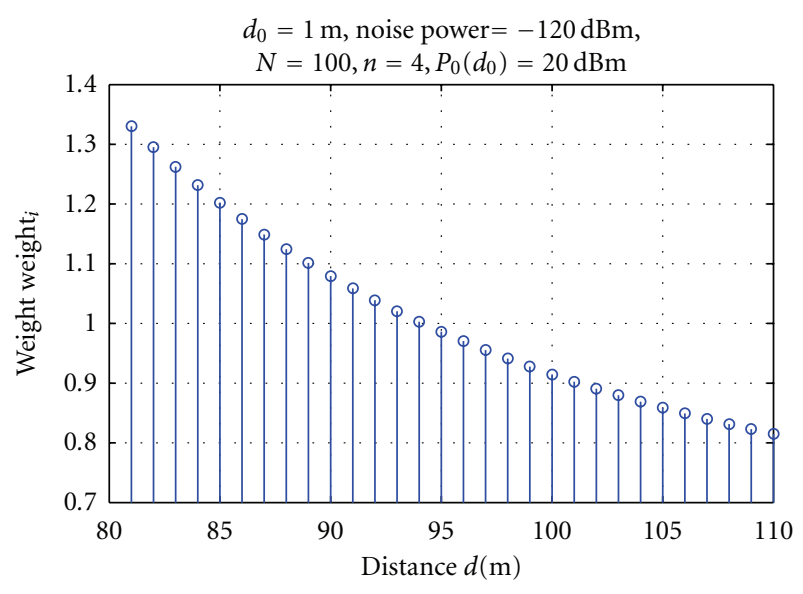

FIGURe 9: Assigned weight for CRs at different distance when weight variance $=0.02$.

future work, we will try to derive the optimal design for the weight assignment.

\section{Acknowledgments}

This paper was supported by AFOSR under Grant FA955009-0630 and by NSF under Grant CNS 0619693.

\section{References}

[1] J. Mitola, Cognitive Radio, Licentiate thesis, KTH, Stockholm, Sweden, 1999.

[2] S. Haykin, "Cognitive radio: brain-empowered wireless communications," IEEE Journal on Selected Areas in Communications, vol. 23, no. 2, pp. 201-220, 2005.

[3] M. A. Sturza and F. Ghazvinian, "White Spaces Engineering Study: can cognitive radio technology operating in the TV white spaces completely protect licensed TV broadcasting?" New America Foundation Wireless Future Program, working paper no.16, January 2007.

[4] K. Watanabe, K. Ishibashi, and R. Kohno, "Performance of cognitive radio technologies in the presence of primary radio systems," in Proceedings of the 18th Annual IEEE International Symposium on Personal, Indoor and Mobile Radio Communications (PIMRC '07), September 2007.

[5] D. Cabric, S. M. Mishra, and R. W. Brodersen, "Implementation issues in spectrum sensing for cognitive radios," in Proceedings of the of the 38th Asilomar Conference on Signals, Systems and Computers, pp. 772-776, November 2004.

[6] F. F. Digham, M.-S. Alouini, and M. K. Simon, "On the energy detection of unknown signals over fading channels," in Proceedings of the International Conference on Communications (ICC '03), vol. 5, pp. 3575-3579, May 2003.

[7] E. C. Y. Peh, Y.-C. Liang, and Y. L. Guan, "Optimization of cooperative sensing in cognitive radio networks: a sensingthroughput tradeoff view," in Proceedings of the IEEE International Conference on Communications (ICC'09), pp. 1-5, June 2009.

[8] G. Ganesan and Y. Li, "Cooperative spectrum sensing in cognitive radio networks," in Proceedings of the 1st IEEE International Symposium on New Frontiers in Dynamic Spectrum Access Networks (DySPAN '05), pp. 137-143, November 2005.
[9] J. N. Laneman, D. N. C. Tse, and G. W. Wornell, "Cooperative diversity in wireless networks: efficient protocols and outage behavior," IEEE Transactions on Information Theory, vol. 50, no. 12, pp. 3062-3080, 2004.

[10] G. Ganesan and Y. Li, "Cooperative spectrum sensing in cognitive radio_-part I: two user networks," IEEE Transactions on Wireless Communications, vol. 6, no. 6, pp. 2204-2213, 2007.

[11] G. Ganesan and Y. Li, "Cooperative spectrum sensing in cognitive radio-part II: multi-user networks," IEEE Transactions on Wireless Communications, vol. 6, no. 6, pp. 2214-2222, 2007.

[12] G. Ganesan and Y. Li, "Agility improvement through cooperative diversity in cognitive radio," in Proceedings of the IEEE Global Telecommunications Conference (GLOBECOM '05), vol. 5, pp. 2505-2509, 2005.

[13] Z. Jiang, X. Zhengguang, W. Furong, H. Benxiong, and Z. Bo, "Double threshold energy detection of cooperative spectrum sensing in cognitive radio," in Proceedings of the $3 \mathrm{rd}$ International Conference on Cognitive Radio Oriented Wireless Networks and Communications (CrownCom '08), pp. 1-5, 2008.

[14] J.-W. Ho, M. Wright, and S. K. Das, "Fast detection of replica node attacks in mobile sensor networks using sequential analysis," in Proceedings of the 28th Conference on Computer Communications (INFOCOM '09), pp. 1773-1781, June 2009.

[15] Z. Quan, S. Cui, and A. H. Sayed, "Optimal linear cooperation for spectrum sensing in cognitive radio networks," IEEE Journal on Selected Topics in Signal Processing, vol. 2, no. 1, pp. 28-40, 2008.

[16] L.-H. He, X.-Z. Xie, X.-T. Dong, and T. Zhou, "Twicecooperative spectrum sensing in cognitive radio systems," in Proceedings of the International Conference on Wireless Communications, Networking and Mobile Computing (WiCOM '08), 2008.

[17] K. B. Letaief and W. Zhang, "Cooperative communications for cognitive radio networks," Proceedings of the IEEE, vol. 97, no. 5, pp. 878-893, 2009.

[18] A. Ghasemi and E. S. Sousa, "Collaborative spectrum sensing in cognitive radio networks," in Proceedings of the 1st IEEE International Symposium on New Frontiers in Dynamic Spectrum Access Networks (DySPAN '05), pp. 131-136, November 2005.

[19] RFC 3561: Ad hoc On-Demand Distance Vector (AODV) Routing, The Internet Society, July 2003.

[20] J. Neyman and E. Pearson, "On the problem of the most efficient tests of statistical hypotheses," Philosophical Transactions of the Royal Society of London, pp. 289-337, 1933.

[21] K. P. Varshney, Distributed Detection and Data Fusion, Springer, New York, NY, USA, 1997.

[22] W. Wenzhong, Z. Weixia, Z. Zheng, and Y. Yabin, "Detection fusion by hierarchy rule for cognitive radio," in Proceedings of the 3rd International Conference on Cognitive Radio Oriented Wireless Networks and Communications (CrownCom '08), pp. $1-5$, May 2008.

[23] W. Zhang, R. K. Mallik, and K. B. Letaief, "Cooperative spectrum sensing optimization in cognitive radio networks," in Proceedings of the IEEE International Conference on Communications (ICC '08), pp. 3411-3415, 2008. 

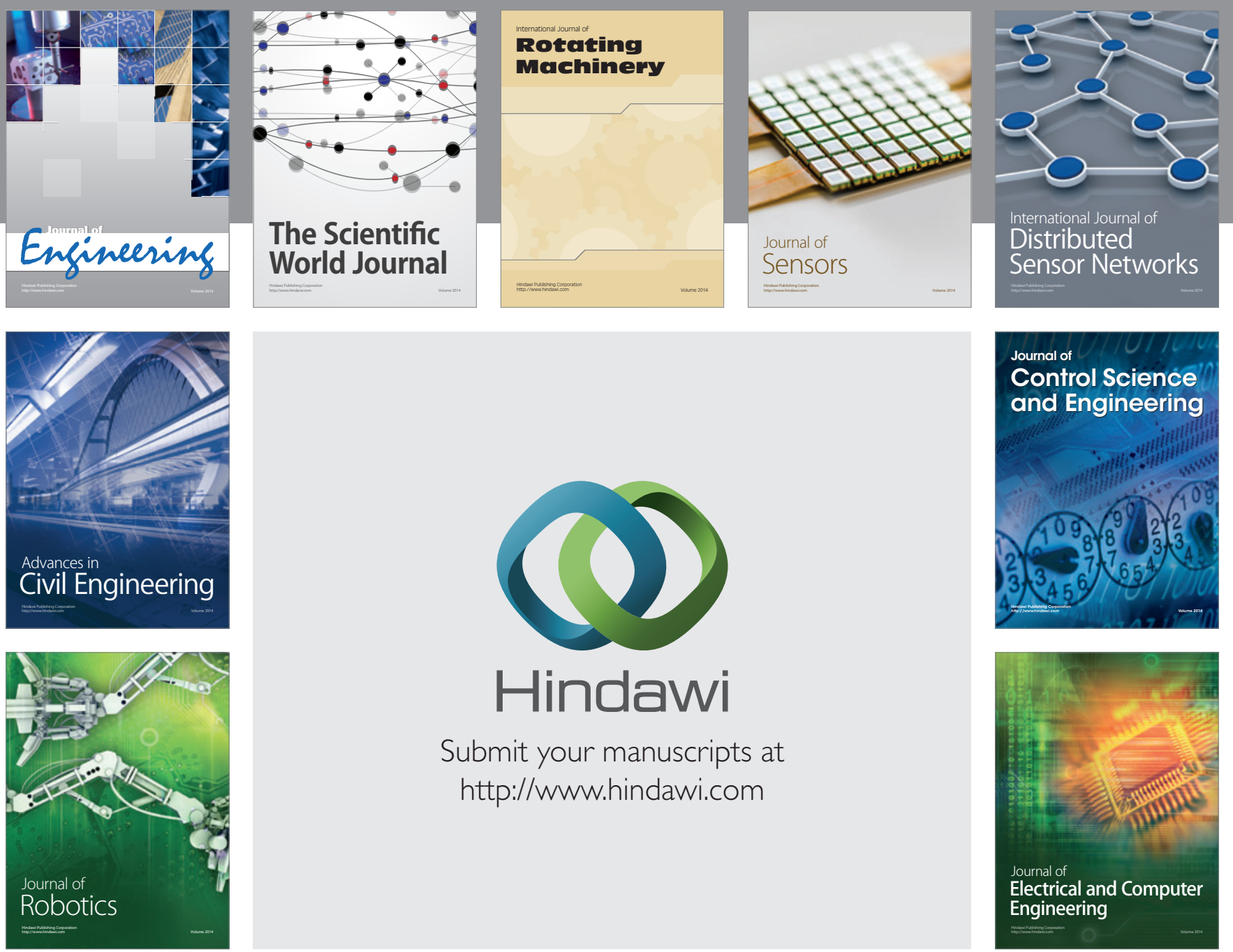

Submit your manuscripts at

http://www.hindawi.com
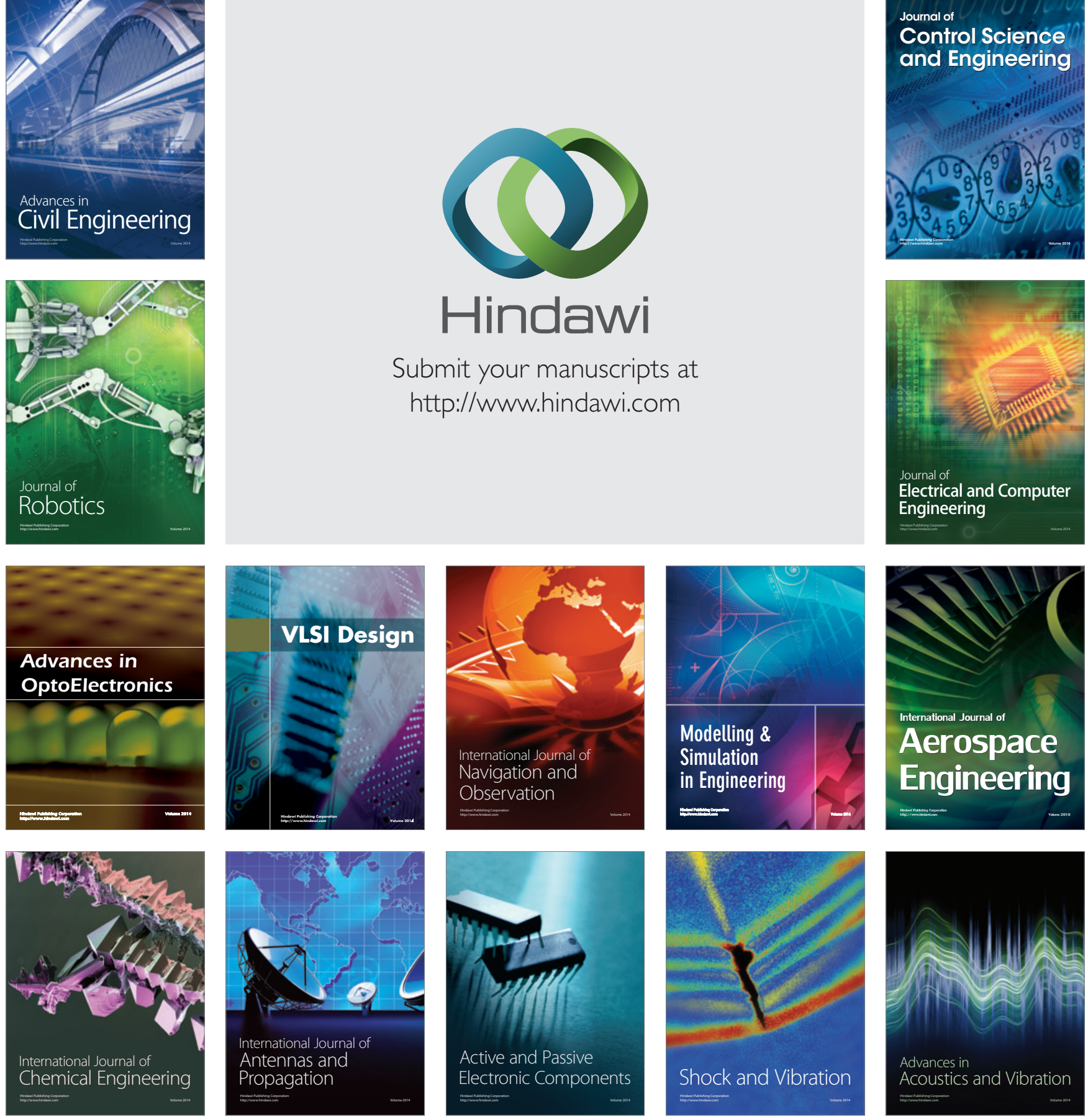